TRANSACTIONS OF THE

AMERICAN MATHEMATICAL SOCIETY

Volume 357, Number 10, Pages 4225-4248

S 0002-9947(04)03658-X

Article electronically published on December 9, 2004

\title{
ELLIPTIC PLANAR VECTOR FIELDS WITH DEGENERACIES
}

\author{
ABDELHAMID MEZIANI
}

\begin{abstract}
This paper deals with the normalization of elliptic vector fields in the plane that degenerate along a simple and closed curve. The associated homogeneous equation $L u=0$ is studied and an application to a degenerate Beltrami equation is given.
\end{abstract}

\section{INTRODUCTION}

This paper deals mainly with the normalization and integrability of a class of smooth complex-valued vector fields in the plane. A vector field $L$ in this class will be assumed to be elliptic throughout except on a closed and simple curve $\Sigma$ along which it is supposed to be tangent and such that $L \wedge \bar{L}$ vanishes to a constant order on $\Sigma$. The questions considered here are those of integrability and normalization of $L$ in a tubular neighborhood of $\Sigma$.

We can assume that $\Sigma$ is the circle $\{0\} \times S^{1} \subset \mathbb{R} \times S^{1}$ and that in a neighborhood of $\Sigma$, the vector field $L$ has the expression

$$
L_{n}=\frac{\partial}{\partial \theta}-i r^{n+1} a(r, \theta) \frac{\partial}{\partial r}
$$

with $\operatorname{Re}(a(0, \theta)) \neq 0$ for every $\theta$. The case $n=0$ is now well understood (see [CG], M1], and [M2]). The focus of this paper is then on the case $n \geq 1$.

To achieve a normal form for $L_{n}$, we construct a $C^{\infty}$-integral of $L_{n}$ in a ring $A_{\delta}=(-\delta, \delta) \times S^{1}$. We use this integral to show that $L_{n}$ is $C^{\infty}$-conjugate to the rotation invariant vector field $R_{n}$ given by

$$
R_{n}=\frac{\partial}{\partial \theta}-i \frac{r^{n+1}}{r P^{\prime}(r)-n P(r)+\mu r^{n}} \frac{\partial}{\partial r}
$$

where $\mu \in \mathbb{C}$ and $P(r)$ is a polynomial with degree at most $n-1$ and such that $\operatorname{Re}(P(0))<0$. The polynomial $P$ and $\mu$ are uniquely determined by the vector field $L_{n}$. It follows, in particular, that two distinct vector fields given by (2) cannot be conjugate. A corresponding $C^{\infty}$-integral of $R_{n}$ is the function

$$
f_{n}(r, \theta)=\exp \left(\epsilon(r)^{n}\left(\frac{P(r)}{r^{n}}+\mu \log |r|+i \theta\right)\right),
$$

with $\epsilon(r)=\frac{r}{|r|}$. Note that since $\operatorname{Re}(P(0))<0, f_{n} \in C^{\infty}\left(A_{\delta}\right)$ (for $\delta$ small enough) and that it vanishes to infinite order along $\Sigma$.

Received by the editors January 13, 2003 and, in revised form, December 23, 2003.

2000 Mathematics Subject Classification. Primary 35F05; Secondary 30G20.

Key words and phrases. Beltrami equation, CR equation, elliptic vector field, normalization. 
Our motivation for seeking such normal forms is in the subsequent study of the pde's related to the structures defined by $L$. The normal forms allow us to write the equations in such a way that they can be analyzed. Related papers about solvability of vector fields near the characteristic set include [BCH], [BCP], BgM1, BgM2, [BhM1, BhM2, M1], [M2, [NT], [T1, T2] and many others (see the extensive list of references contained in [T2]).

The organization of this paper is as follows. In Section 1, we set the preliminaries and recall the main results of [CG], M1] and [M2] about the case $n=0$. In Section 2 , we construct a unique series that is a formal solution of the equation $L_{n} u=0$. The series has the form

$$
\frac{P(r)}{r^{n}}+\mu \log |r|+i \theta+\sum_{j=1}^{\infty} f_{j}(\theta) r^{j},
$$

with $\mu \in \mathbb{C}, P$ a polynomial with degree $\leq n-1$, and $f_{j} \in C^{\infty}\left(S^{1}\right)$ (or in $C^{\omega}\left(S^{1}\right)$ when $L$ is real analytic). In general, the series $\sum f_{j}(\theta) r^{j}$ appearing in (4) diverges for every $r \neq 0$. This is illustrated by an example in Section 3. In order to construct a nonconstant $C^{\infty}$ solution of $L_{n} u=0$, we study, in Section 4, particular $\mathrm{CR}$ equations. Namely, equations of the form

$$
\frac{\partial w}{\partial \bar{z}}=\frac{f(z)}{z} \quad \text { and } \quad \frac{\partial w}{\partial \bar{z}}=\mu(z) \frac{\partial w}{\partial z}
$$

where the coefficients $f(z)$ and $\mu(z)$ are functions of order $o\left(\log ^{-q} \frac{1}{|z|}\right)$ for every $q>0$. In Section 5, we use the series (4) and results obtained in Section 4 to construct a $C^{\infty}$-integral for $L_{n}$. The normal form (3) for $L_{n}$ is obtained in Section 6. The kernel of the operator $R_{n}$ is studied in Section 7. We prove that all $C^{0}$ solutions of $R_{n} u=0$, in a neighborhood of the circle $r=0$, are $C^{\infty}$ functions. This result does not have a local counterpart. Indeed, for every $p \in \Sigma$, the equation $R_{n} u=0$ has continuous solutions defined in a neighborhood of $p$ that are not $C^{\infty}$. For the distribution solutions, we show that if $u \in \mathcal{D}^{\prime}\left(A_{\delta}\right)$ solves $R_{n} u=0$ and has support in $\Sigma$, then there are constants $c_{0}, \cdots, c_{n-1}$ such that

$$
\langle u, \phi\rangle=\sum_{j=0}^{n-1} c_{j} \int_{0}^{2 \pi} \frac{\partial^{j} \phi}{\partial r^{j}}(0, \theta) d \theta, \quad \forall \phi \in \mathcal{D}\left(A_{\delta}\right) .
$$

In the last section we make use of the normalization of the vector $L_{0}$ to study a degenerate Beltrami equation.

\section{PREliminaries AND FIRST ORDER CASE}

In this section, we give the preliminary settings and recall the normalization for the case $n=0$. Let

$$
L=a(x, y) \frac{\partial}{\partial x}+b(x, y) \frac{\partial}{\partial y}
$$

be a vector field in $\mathbb{R}^{2}$. We assume that the coefficients $a$ and $b$, are $C^{\infty}$ or $C^{\omega}$ functions, are $\mathbb{C}$-valued and that they do not vanish simultaneously. Let $\bar{L}$ be the complex conjugate vector field

$$
\bar{L}=\bar{a} \frac{\partial}{\partial x}+\bar{b} d y
$$


The vector field $L$ is said to be elliptic at a point $p$ if $L$ and $\bar{L}$ are independent at $p$. If $L$ is elliptic at each point of an open set $\Omega$, then it is equivalent in $\Omega$ to the $\mathrm{CR}$ vector field

$$
\frac{\partial}{\partial \bar{z}}=\frac{1}{2} \frac{\partial}{\partial x}+\frac{i}{2} \frac{\partial}{\partial y}
$$

Denote by $\Sigma$ the characteristic set of $L$. That is, the set of points where $L$ fails to be elliptic:

$$
\Sigma=\left\{p \in \mathbb{R}^{2} ; L \text { and } \bar{L} \text { are independent }\right\}
$$

We make the following assumptions

(H1) $\Sigma$ is a simple and closed curve;

(H2) $L$ is tangent to $\Sigma$ at each point $p \in \Sigma$;

(H3) $L \wedge \bar{L}$ vanishes to a constant order $n+1$ along $\Sigma$.

It follows from the local representation of such vector fields (see [T1] or [T2]) that for each given $p \in \Sigma$, there are coordinates $(s, t)$, centered at $p$, such that in a neighborhood of the point $p$, the vector field $L$ is a multiple of

$$
\frac{\partial}{\partial t}-i s^{n+1} \alpha(s, t) \frac{\partial}{\partial s}
$$

some real-valued function $\alpha$ satisfying $\alpha(0) \neq 0$. It follows at once, that $L$ satisfies the Nirenberg-Treves condition $(P)$ at each point on $\Sigma$ (see [NT] or [T1] or [T2]). These vector fields are thus locally integrable and locally solvable. In fact, the function $\alpha$ of (1.4) can be assumed to be identically equal to 1 (see [CG]). Thus, a vector field $L$ satisfying hypotheses (H1), (H2), and (H3) can be viewed as follows: in a neighborhood of a point $p \notin \Sigma, L$ is equivalent to $\frac{\partial}{\partial \bar{z}}$ and in a neighborhood of a point $p \in \Sigma, L$ is equivalent to $\frac{\partial}{\partial y}-i x^{n+1} \frac{\partial}{\partial x}$. These vector fields are therefore well understood when viewed locally. Their global behavior is, however, more complicated. Our aim here is to obtain normal forms for $L$ in a tubular neighborhood of the characteristic set $\Sigma$.

From the assumption (H1), we can assume that $\Sigma$ is a circle, that $L$ is defined in $\mathbb{R} \times S^{1}$, and that

$$
\Sigma=\{0\} \times S^{1}
$$

Let

$$
L=\alpha(r, \theta) \frac{\partial}{\partial \theta}+\beta(r, \theta) \frac{\partial}{\partial r},
$$

where $(r, \theta)$ are the coordinates in $\mathbb{R} \times S^{1}$. It follows from hypotheses $(\mathrm{H} 2)$ and (H3) that there exists $\delta>0$ such that in the ring

$$
A_{\delta}=(-\delta, \delta) \times S^{1}
$$

the vector field $L$ is a multiple of a vector field $L_{n}$ of the form

$$
L_{n}=\frac{\partial}{\partial \theta}-i r^{n+1} a(r, \theta) \frac{\partial}{\partial r}
$$

for some $a \in C^{\infty}\left(A_{\delta}\right)$ satisfying $\operatorname{Re}(a(r, \theta)) \neq 0$ for every $(r, \theta) \in A_{\delta}$. Without loss of generality, we can assume that

$$
R e(a(r, \theta))>0 \quad \forall(r, \theta) \in A_{\delta} .
$$


The linear case $n=0$ was studied in [M1] and [M2] and the study was completed in [CG]. It is proved in [M1] and [M2] that the complex number

$$
\lambda=\frac{1}{2 \pi} \int_{0}^{2 \pi} a(0, \theta) d \theta \in \mathbb{R}^{+}+i \mathbb{R}
$$

is an invariant that characterizes $L_{0}$. It is shown in [M2] that if $\operatorname{Im} \lambda \neq 0$, then for every $k \in \mathbb{Z}^{+}$, there exists a $C^{k}$-diffeomorphism of $A_{\delta}$ that transforms $L_{0}$ to a multiple of the vector field

$$
T_{\lambda}=\frac{\partial}{\partial \theta}-i r \lambda \frac{\partial}{\partial r} .
$$

When $\operatorname{Im} \lambda=0$ (i.e., $\lambda \in \mathbb{R}^{+}$), it is also proved in [M2] that $L_{0}$ is equivalent to $T_{\lambda}$ but only under a $C^{1+\sigma}$-diffeomorphism for some $0<\sigma<1$. In [CG], the above result about $C^{k}$ equivalence is extended to include the case $\lambda \in \mathbb{R} \backslash \mathbb{Q}$.

In the real analytic category, it is proved in [M1] that $L_{0}$ is $C^{\omega}$-equivalent to $T_{\lambda}$, if the equation $L_{0} u=0$ has a nonconstant $C^{\omega}$ solution. This is equivalent to saying that the holonomy group of $\Sigma$ is periodic. It is proved in $[\mathrm{CG}$. that, when $\lambda \in \mathbb{R} \backslash \mathbb{Q}$ and $\lambda$ satisfies a certain diophantine condition (Bruno condition), the vector field $L_{0}$ is $C^{\omega}$-equivalent to $T_{\lambda}$. It is also proved that there are $C^{\omega}$ vector fields $L_{0}$ with $\lambda \in \mathbb{R}$ not satisfying the Bruno condition such that $L_{0}$ is not $C^{\omega}$ equivalent to $T_{\lambda}$.

\section{Formal integrability}

We show that a vector field $L_{n}$ as in (1.8) has a formal integral. First, we rewrite the vector field in more suitable coordinates.

Lemma 2.1. There is a $C^{\infty}$ change of coordinates that transforms $L_{n}$ into a multiple of

$$
\frac{\partial}{\partial \theta}-i r^{n+1}\left(c_{0}+c(r, \theta)\right) \frac{\partial}{\partial r}
$$

where $c_{0}=1+i \beta \in \mathbb{C}$, and $c(r, \theta) \in C^{\infty}\left(A_{\delta}\right)$ satisfying $c(0, \theta) \equiv 0$. (The change of coordinates is $C^{\omega}$ when $L$ is $C^{\omega}$.)

Proof. With $L_{n}$ as in (1.8), consider the 1-form $\omega$ given by

$$
\omega=d r+i r^{n+1} a(r, \theta) d \theta .
$$

With our assumption $\operatorname{Rea}(r, \theta)>0$, we have

$$
\lambda=\frac{1}{2 \pi} \int_{0}^{2 \pi} a(0, \theta) d \theta=a+i b \in \mathbb{R}^{+}+i \mathbb{R} .
$$

Since $n>0$, we can replace $r$ by $r_{1}=\sqrt[n]{a} r$ in such a way that in the new coordinates $\left(r_{1}, \theta\right)$ we have $\operatorname{Re}(\lambda)=1$. Hence, from now on we can assume that

$$
a(r, \theta)=1+i b_{0}+\gamma_{1}(\theta)+i \gamma_{2}(\theta)+r a_{1}(r, \theta),
$$

where $b_{0} \in \mathbb{R}, a_{1} \in C^{\infty}\left(A_{\delta}\right)$, and $\gamma_{1}, \gamma_{2} \in C^{\infty}\left(S^{1}\right)$ are $\mathbb{R}$-valued and have averages on $S^{1}$ equal to 0 , i.e.,

$$
\int_{0}^{2 \pi} \gamma_{k}(\theta) d \theta=0, \quad k=1,2 .
$$

Consider the new angle $\phi$ defined by

$$
\phi(\theta)=\theta+\int_{0}^{\theta} \gamma_{1}(s) d s .
$$


It follows from the hypothesis on $a$ that $\phi^{\prime}(\theta)>0$ and from (2.5) that $\phi(\theta+2 \pi)=$ $\phi(\theta)+2 \pi$. With respect to the coordinates $(r, \phi)$, the form $\omega$ has the expression

$$
\omega=d r+i r^{n+1}(1+i \beta-i \chi(\phi)+O(r)) d \phi,
$$

with $\beta \in \mathbb{R}, \chi \in C^{\infty}\left(S^{1}\right)$, real valued and with zero average on $S^{1}$. Consider the new variables $(\rho, \phi)$ in $A_{\delta}$, where

$$
\rho=\frac{r}{\sqrt[n]{1-n r^{n} m(\phi)}} \quad \text { with } m(\phi)=\int_{0}^{\phi} \chi(s) d s .
$$

A calculation shows that

$$
d r+r^{n+1} \chi(\phi)=\frac{d \rho}{{\sqrt[n]{1+n \rho^{n} m(\phi)}}^{n+1}} .
$$

In the $(\rho, \phi)$ coordinates, the form $\omega$ is a multiple of

$$
d \rho+i \rho^{n+1}(1+i \beta+O(\rho)) d \phi .
$$

Consequently, $L_{n}$ is a multiple of a vector field given by (2.1).

From now on, we will assume that $L_{n}$ is given by (2.1). We will show that $L_{n}$ has a formal first integral. More precisely, we have the following proposition.

Proposition 2.1. Let $L_{n}$ be as in (2.1). Then there exist unique constants $\mu \in \mathbb{C}$, $\alpha_{-n}, \cdots, \alpha_{-1} \in \mathbb{C}$ and a unique sequence of functions $f_{j}(\theta) \in C^{\infty}\left(S^{1}\right), j \in \mathbb{Z}^{+}$, such that the series

$$
f(r, \theta)=\frac{\alpha_{-n}}{r^{n}}+\cdots+\frac{\alpha_{-1}}{r}+\mu \log |r|+i \theta+\sum_{j=1}^{\infty} f_{j}(\theta) r^{j}
$$

solves formally the equation $L_{n} f=0$.

Remark 2.1. By a formal solution of the equation $L_{n} u=0$, we mean the following. For each $N \in \mathbb{Z}^{+}$, the function $f_{N}$ defined by

$$
f_{N}(r, \theta)=\frac{\alpha_{-n}}{r^{n}}+\cdots+\frac{\alpha_{-1}}{r}+\mu \log |r|+i \theta+\sum_{j=1}^{N} f_{j}(\theta) r^{j}
$$

satisfies $L_{n} f_{N}=o\left(r^{N}\right)$.

Remark 2.2. When $L_{n}$ is real analytic, the functions $f_{j}(\theta) \in C^{\omega}\left(S^{1}\right)$.

Proof of Proposition 2.1. The Taylor expansion of the coefficient of $L_{n}$ given by (2.1) is

$$
T_{0}\left(c_{0}+c(r, \theta)\right)=\sum_{j=0}^{\infty} c_{j}(\theta) r^{j}
$$

with $c_{j}(\theta)=\frac{1}{j !} \frac{\partial^{j} c}{\partial r^{j}}(0, \theta)$. We write

$$
c_{j}(\theta)=c_{j}^{0}+\gamma_{j}(\theta)
$$

where

$$
c_{j}^{0}=\frac{1}{2 \pi} \int_{0}^{2 \pi} c_{j}(\theta) d \theta
$$


Note that $c_{0}=1+i \beta, \gamma_{0}=0$, and that since for $j \geq 1$, the average of $\gamma_{j}(\theta)$ on $S^{1}$ is zero, then

$$
\int_{0}^{\theta} \gamma_{j}(s) d s \in C^{\infty}\left(S^{1}\right)
$$

In order for the series (2.9) to formally satisfy $L_{n} u=0$, we need to have

$$
\begin{aligned}
i+\sum_{j=1}^{\infty} f_{j}^{\prime}(\theta) r^{j}-i r^{n+1} \sum_{l=0}^{\infty}\left(c_{l}^{0}+\right. & \left.\gamma_{l}(\theta)\right) r^{l}\left[\frac{-n \alpha_{-n}}{r^{n+1}}+\cdots+\frac{-\alpha_{-1}}{r^{2}}\right. \\
\left.+\frac{\mu}{r}+\sum_{j=1}^{\infty} j f_{j}(\theta) r^{j-1}\right] & =0 .
\end{aligned}
$$

After grouping like terms and equating to zero the coefficient of $r^{m}$, we obtain the following equations:

$$
\begin{aligned}
& 1+n \alpha_{-n} c_{0}=0, \quad \text { for } m=0 ; \\
& f_{m}^{\prime}+\sum_{k=n-m}^{n} i k \alpha_{-k}\left(c_{m-n+k}^{0}+\gamma_{m-n+k}\right)=0, \quad \text { for } m=1, \cdots, n-1 ; \\
& f_{n}^{\prime}-i c_{0} \mu+\sum_{k=1}^{n} i k \alpha_{-k}\left(c_{k}^{0}+\gamma_{k}\right)=0, \quad \text { for } m=n ; \\
& f_{m}^{\prime}-i \mu\left(c_{m-n}^{0}+\gamma_{m-n}\right)+\sum_{k=1}^{n}\left(c_{m-n+k}^{0}+\gamma_{m-n+k}\right) \\
& -\sum_{k=1}^{m-n} i k f_{k}\left(c_{m-n-k}^{0}+\gamma_{m-n-k}\right)=0 \quad \text { for } m \geq n+1 .
\end{aligned}
$$

It follows from (2.16) that

$$
\alpha_{-n}=\frac{-1}{n c_{0}}
$$

is uniquely determined. We set $m=1$ in (2.17) to obtain

$$
f^{\prime}(\theta)=-i\left((n-1) \alpha_{-(n-1)} c_{0}+n \alpha_{-n}\left(c_{1}^{0}+\gamma_{1}(\theta)\right)\right) .
$$

It follows from (2.14) that this equation has a $2 \pi$-periodic solution $f_{1}(\theta)$ if and only if

$$
(n-1) c_{0} \alpha_{-(n-1)}+n \alpha_{-n} c_{1}^{0}=0 .
$$

This determines $\alpha_{-(n-1)}$ uniquely. For this value of $\alpha_{-(n-1)}$, the function $f_{1} \in$ $C^{\infty}\left(S^{1}\right)$ is determined up to an additive constant of integration $K_{1}$. By induction, suppose that there are unique constants $\alpha_{-n}, \cdots, \alpha_{n-l}$, with $l<n-1$ so that the differential equations in (2.17) for $m=1, \cdots, l$ have $2 \pi$-periodic solutions $f_{1}, \cdots, f_{l}$ that are determined up to additive constants $K_{1}, \cdots, K_{l}$. For $m=l+1$, we obtain the equation

$$
\begin{aligned}
f_{l+1}^{\prime}(\theta)= & -i(n-(l+1)) \alpha_{-(n-(l+1))} c_{0} \\
& -\sum_{k=n-l}^{n} i k \alpha_{-k}\left(c_{l+1-n+k}^{0}+\gamma_{l+1-n+k}(\theta)\right) .
\end{aligned}
$$


It follows from (2.14) that equation (2.23) has a $2 \pi$-periodic solution $f_{l+1}$ (determined up to an additive constant) for the unique value of $\alpha_{-(n-(l+1))}$ given by

$$
(n-(l+1)) c_{0} \alpha_{-(n-(l+1))}+\sum_{k=n-l}^{n} k \alpha_{-k} c_{l+1-n+k}^{0}=0 .
$$

This shows that there are unique constants $\alpha_{-n}, \cdots, \alpha_{-1}$ so that equations (2.17) have $2 \pi$-periodic solutions $f_{1}, \cdots, f_{n-1}$ that are determined up to additive constants.

Now that $\alpha_{-n}, \cdots, \alpha_{-1}$ are determined, there is a unique constant $\mu$ given by

$$
-c_{0} \mu+\sum_{k=1}^{n} k \alpha_{-k} c_{k}^{0}=0
$$

so that the equation (2.18) has a $2 \pi$-periodic solution $f_{n}(\theta)$.

For $m=n+1$, equation (2.19) has a $2 \pi$-periodic solution $f_{n+1}(\theta)$ if and only if

$$
\int_{0}^{2 \pi}\left(\mu c_{1}^{0}+\sum_{k=1}^{n} k \alpha_{-k} c_{1+k}^{0}-f_{1}(\theta) c_{0}\right) d \theta=0 .
$$

There is a unique choice of the constant $K_{1}$ for which equation (2.26) holds. For this choice of $K_{1}$ (so $f_{1}$ is now uniquely determined), $f_{n+1}$ is determined up to an additive constant. By induction, suppose the functions $f_{1}, \cdots, f_{l}$ are uniquely determined so that equations (2.19) have $2 \pi$-periodic solutions $f_{n+1}, \cdots, f_{n+l}$ determined up to additive constants. The equation for $m=n+l+1$ has a $2 \pi$-periodic solution $f_{n+l+1}$ if and only if

$$
\begin{aligned}
(l+1) c_{0} \int_{0}^{2 \pi} f_{l+1}(\theta) d \theta=\int_{0}^{2 \pi} & \left(-\mu c_{l+1}^{0}+\sum_{k=1}^{n} k \alpha_{-k} c_{l+1+k}^{0}\right) d \theta \\
& +\sum_{k=1}^{l} \int_{0}^{2 \pi} k f_{k}(\theta)\left(c_{l+1-k}^{0}+\gamma_{l+1-k}(\theta)\right) d \theta .
\end{aligned}
$$

There is a unique constant $K_{l+1}$ (so $f_{l+1}$ is uniquely determined) for which (2.28) holds. This completes the proof of the proposition.

\section{An EXAmple}

We give an example of a real analytic vector field with $n=1$ for which the series solution constructed in the previous section diverges for every $r \neq 0$. Consider the vector field

$$
L_{1}=\frac{\partial}{\partial \theta}-i r^{2}\left(1+r \mathrm{e}^{i \theta}\right) \frac{\partial}{\partial r} .
$$

We have the following proposition.

Proposition 3.1. For the vector field $L_{1}$ of (3.1), the series

$$
f(r, \theta)=\frac{\alpha_{-1}}{r}+\mu \log |r|+i \theta+\sum_{j=1}^{\infty} f_{j}(\theta) r^{j},
$$


with $f_{j} \in C^{\infty}\left(S^{1}\right)$, solves formally $L_{1} u=0$, if and only if

$$
\begin{gathered}
\alpha_{-1}=-1, \quad \mu=0, \quad f_{1}(\theta)=e^{i \theta} \quad \text { and } \\
f_{j}(\theta)=(j-1) ! e^{i \theta}+\sum_{k=2}^{j-1} f_{j k} e^{i k \theta}, \quad \text { for } j=2,3, \cdots,
\end{gathered}
$$

where $f_{j k}$ are constants. Consequently, the series $\sum_{j=1}^{\infty} f_{j}(\theta) r^{j}$ diverges for every $r \neq 0$.

Proof. It follows at once from $L_{1} u=0$ that

$$
\begin{aligned}
i\left(1+\alpha_{-1}\right)+ & \left(f_{1}^{\prime}+i \alpha_{-1} \mathrm{e}^{i \theta}-i \mu\right) r+\left(f_{2}^{\prime}-i \mu \mathrm{e}^{i \theta}-i f_{1}\right) r^{2} \\
& +\sum_{m \geq 3}^{\infty}\left(f_{m}^{\prime}-i(m-1) f_{m-1}-i(m-2) \mathrm{e}^{i \theta} f_{m-2}\right) r^{m}=0 .
\end{aligned}
$$

Hence $\alpha_{-1}=-1$ and then

$$
f_{1}^{\prime}(\theta)-i \mathrm{e}^{i \theta}-i \mu=0
$$

has a $2 \pi$-periodic solution only when $\mu=0$. In this case

$$
f_{1}(\theta)=\mathrm{e}^{i \theta}+K_{1} \text {. }
$$

By equating the coefficient of $r^{2}$ to 0 , we get

$$
f_{2}^{\prime}(\theta)=i f_{1}(\theta)=i \mathrm{e}^{i \theta}+i K_{1} .
$$

This equation has a $2 \pi$-periodic solution if $K_{1}=0$ and then

$$
f_{2}(\theta)=\mathrm{e}^{i \theta}+K_{2} .
$$

By equating the coefficient of $r^{3}$ to zero, we see that $f_{3}$ exists only when $K_{2}=0$ and then

$$
f_{3}(\theta)=2 \mathrm{e}^{i \theta}+\frac{1}{2} \mathrm{e}^{2 i \theta}+K_{3} .
$$

By induction, suppose that $f_{j}$ has the expression given in (3.3) for $j=1, \cdots, m-1$, then it follows from (3.4) that

$$
\begin{aligned}
f_{m}^{\prime}(\theta) & =(m-1) i f_{m-1}(\theta)+(m-2) i \mathrm{e}^{i \theta} f_{m-2}(\theta) \\
& =(m-1) ! i \mathrm{e}^{i \theta}+\sum_{k=2}^{m-1} d_{m k} \mathrm{e}^{i k \theta}
\end{aligned}
$$

with $d_{m k}$ constants. Expression (3.3) for $f_{m}$ follows at once.

To complete the proof of the proposition, observe that if $\sum f_{j}(\theta) r^{j}$ has positive radius of convergence, then the function

$$
M(r)=\frac{1}{2 \pi} \int_{0}^{2 \pi}\left(\sum_{j=1}^{\infty} f_{j}(\theta) r^{j}\right) \mathrm{e}^{-i \theta} d \theta
$$

would be real analytic at $r=0$. But it follows from (3.3) that

$$
M(r)=\sum_{j=1}^{\infty}(j-1) ! r^{j}
$$

with radius of convergence equal to zero. 


\section{Some Results about the CR operator}

We will prove some results about the CR equation that will be needed to construct a $C^{\infty}$ integral for $L_{n}$. Consider the space of functions defined in the disc $D(0, R) \subset \mathbb{C}$ by

$$
E_{R}=\left\{f \in C^{\infty}(\overline{D(0, R)} \backslash 0) ; f(z)=o\left(\log ^{-q} \frac{1}{|z|}\right) \quad \forall q>0\right\} .
$$

Lemma 4.1. Let $f \in E_{R}$ and let

$$
g(z)=\iint_{D(0, R)} \frac{f(\zeta)}{\zeta^{2}(\zeta-z)} d \xi d \eta
$$

where $\zeta=\xi+i \eta$. Then there is $R_{1}<R$ such that $z g(z) \in E_{R_{1}}$.

Proof. Since $f \in E_{R}$, then it is not difficult to see that $g$ is $C^{\infty}$ for $z \neq 0$. We need only to show that for a given $q>0, z g(z)=o\left(\log ^{-q} \frac{1}{|z|}\right)$. Let

$$
D(0, R)=\Delta_{1} \cup \Delta_{2} \cup \Delta_{3} \cup \Delta_{4},
$$

where

$$
\begin{gathered}
\Delta_{1}=D\left(0, \frac{|z|}{4}\right), \quad \Delta_{2}=D\left(z, \frac{|z|}{4}\right), \\
\Delta_{3}=\left\{\zeta: \frac{|z|}{4}<|\zeta-z|<\frac{|z|}{4} \log ^{q+1} \frac{1}{|z|} \text { and }|\zeta|>\frac{|z|}{4}\right\}, \\
\Delta_{4}=\left\{\zeta: \frac{|z|}{4}<|\zeta|<R \text { and }|\zeta-z|>\frac{|z|}{4} \log ^{q+1} \frac{1}{|z|}\right\} .
\end{gathered}
$$

We have

$$
\begin{aligned}
& |z g(z)| \leq|z| I_{1}+|z| I_{2}+|z| I_{3}+|z| I_{4} \quad \text { with } \\
& I_{j}=\iint_{\Delta_{j}} \frac{|f(\zeta)|}{|\zeta|^{2}|\zeta-z|} d \xi d \eta \quad j=1,2,3,4 .
\end{aligned}
$$

To prove the lemma, we need only to show that

$$
\lim _{|z| \rightarrow 0}|z| I_{j} \log ^{q} \frac{1}{|z|}=0 \quad \text { for } j=1,2,3,4 .
$$

For $\zeta \in \Delta_{1}$, we have $|\zeta-z|>|z|-|\zeta|>\frac{3}{4}|z|$ and so

$$
I_{1} \leq \frac{4}{3|z|} \iint_{\Delta_{1}} \frac{|f(\zeta)|}{|\zeta|^{2}} d \xi d \eta
$$

Since $f \in E_{R}$, then for every $s>0$ there exists $C_{s}>0$ such that

$$
|f(\zeta)| \leq C_{s} \log ^{-s} \frac{1}{|\zeta|}, \quad \forall \zeta \in D(0, R) .
$$

Hence,

$$
\begin{aligned}
I_{1} & \leq \frac{4 C_{q+2}}{3|z|} \iint_{\Delta_{1}} \frac{d \xi d \eta}{|\zeta|^{2} \log ^{q+2} \frac{1}{|\zeta|}} \\
& \leq \frac{8 \pi C_{q+2}}{3|z|} \int_{0}^{|z| / 4} \frac{d r}{r \log ^{q+2} \frac{1}{r}}=\frac{8 \pi C_{q+2}}{3|z|(q+1)} \log ^{-(q+1)} \frac{1}{|z|}
\end{aligned}
$$

and (4.6) holds for $j=1$. 
For $\zeta \in \Delta_{2}$, we have $\frac{|z|}{4}<|\zeta|<\frac{5}{4}|z|$. Thus,

$$
\frac{1}{|\zeta|^{2}}<\frac{16}{|z|^{2}} \quad \text { and } \quad \log ^{-1} \frac{1}{|\zeta|}<\log ^{-1} \frac{4}{5|z|} .
$$

It follows that

$$
\begin{aligned}
I_{2} & \leq \frac{16 C_{q+1}}{|z|^{2}}\left(\log ^{-(q+1)} \frac{4}{5|z|}\right) \iint_{\Delta_{2}} \frac{d \xi d \eta}{|\zeta-z|} \\
& \leq \frac{8 \pi C_{q+1}}{|z|} \log ^{-(q+1)} \frac{4}{5|z|}
\end{aligned}
$$

and (4.6) holds for $j=2$.

For $\zeta \in \Delta_{3}$, we have

$$
\frac{1}{|\zeta|^{2}} \leq \frac{16}{|z|^{2}}
$$

We also have

$$
|\zeta| \leq|z|+|\zeta-z| \leq|z|\left(1+\frac{1}{4} \log ^{q} \frac{1}{|z|}\right) \leq \sqrt{|z|}
$$

(we are assuming $|z|$ small). Thus

$$
\log ^{-1} \frac{1}{|\zeta|} \leq 2 \log ^{-1} \frac{1}{|z|}
$$

and

$$
\begin{aligned}
I_{3} & \leq C_{2 q+1} \iint_{\Delta_{3}} \frac{1}{|\zeta|^{2}|\zeta-z|}\left(\log ^{-(2 q+1)} \frac{1}{|\zeta|}\right) d \xi d \eta \\
& \leq \frac{16 C_{2 q+1}}{|z|^{2}} 2^{2 q+1}\left(\log ^{-(2 q+1)} \frac{1}{|z|}\right) \iint_{\Delta_{3}} \frac{d \xi d \eta}{|\zeta-z|} .
\end{aligned}
$$

Using polar coordinates in the last integral, we have

$$
\iint_{\Delta_{3}} \frac{d \xi d \eta}{|\zeta-z|} \leq 2 \pi \int_{|z| / 4}^{(|z| / 4) \log ^{q} \frac{1}{|z|}} d r=\frac{|z|}{4}\left(\log ^{q} \frac{1}{|z|}-1\right) .
$$

Therefore,

$$
I_{3} \leq \frac{8 \pi C_{2 q+1} 2^{2 q+1}}{|z|}\left(\log ^{-(2 q+1)} \frac{1}{|z|}\right)\left(\log ^{q} \frac{1}{|z|}-1\right)
$$

and (4.6) holds for $j=3$.

Finally, for $\zeta \in \Delta_{4}$, we use

$$
|\zeta-z|>\frac{|z|}{4} \log ^{q+1} \frac{1}{|z|}
$$

to obtain

$$
I_{4} \leq \frac{4}{|z|}\left(\log ^{-(q+1)} \frac{1}{|z|}\right) \iint_{\Delta_{4}} \frac{|f(\zeta)|}{|\zeta|^{2}} d \xi d \eta \leq \frac{4 B}{|z|}\left(\log ^{-(q+1)} \frac{1}{|z|}\right),
$$

where

$$
B=\iint_{D(0, R)} \frac{|f(\zeta)|}{|\zeta|^{2}} d \xi d \eta<\infty .
$$

Therefore (4.6) holds for $j=4$ and the lemma is proved. 
Theorem 4.1. Let $f \in E_{R}$. Then the $C R$ equation

$$
\frac{\partial w}{\partial \bar{z}}=\frac{f(z)}{z}
$$

has a solution $w \in E_{R}$.

Remark 4.1. Note that, in general, for $f \in E_{R}$, the function $\frac{f}{z} \in L^{2}$ but $\frac{f}{z} \notin L^{p}$ for any $p>2$. Hence the classical results about the solvability of the inhomogeneous $\mathrm{CR}$ equation cannot be applied here.

Proof of Theorem 4.1. The function

$$
v(z)=\frac{-1}{\pi} \iint_{D(0, R)} \frac{f(\zeta)}{\zeta-z} d \xi d \eta \in C^{\infty}(D(0, R) \backslash 0) \cap C^{1}(D(0, R)) .
$$

That $v$ is in $C^{\infty}(D(0, R) \backslash 0) \cap C^{\sigma}(D(0, R))$ for any $0<\sigma<1$ follows from classical theory (see [V], Chapter 1); that $v$ is $C^{1}$ at 0 follows from a result of [B], Chapter 2. We have

$$
\frac{\partial v}{\partial z}(z)=\frac{-1}{\pi} \iint_{D(0, R)} \frac{f(\zeta)}{(\zeta-z)^{2}} d \xi d \eta .
$$

Let

$$
u(z)=v(z)-v(0)-\frac{\partial v}{\partial z}(0) z=\frac{-z^{2}}{\pi} \iint_{D(0, R)} \frac{f(\zeta)}{\zeta^{2}(\zeta-z)} d \xi d \eta .
$$

The function $u$ solves

$$
\frac{\partial u}{\partial \bar{z}}=\frac{\partial v}{\partial \bar{z}}=f(z)
$$

Therefore, it follows from Lemma 4.1 and from (4.24) that the function

$$
w(z)=\frac{u(z)}{z} \in E_{R}
$$

and solves equation (4.20).

Lemma 4.2. Let $f \in E_{R}$. The function

$$
P f(z)=\iint_{D(0, R)} \frac{f(\zeta)}{(\zeta-z)^{2}} d \xi d \eta,
$$

where the singular integral is understood in the sense of the Cauchy principal value, satisfies

$$
P f(z)-P f(0) \in E_{R} .
$$

Proof. We know that if $f \in E_{R}$, then $P f$ is $C^{\infty}$ away from 0 (see [V], Chapter 1 ). To prove the lemma, we need only to show that for a given $q>0$,

$$
\lim _{z \rightarrow 0}(P f(z)-P f(0)) \log ^{q} \frac{1}{|z|}=0 .
$$

We can rewrite (see $[\mathrm{V}]$, page 58 )

$$
\begin{aligned}
P f(z)-P f(0)=-z \iint_{D(0, R)} & \frac{f(\zeta)-f(z)}{(\zeta-z)^{2} \zeta} d \xi d \eta \\
& -z \iint_{D(0, R)} \frac{f(\zeta)}{\zeta^{2}(\zeta-z)} d \xi d \eta-\pi f(z) \frac{\bar{z}}{z}
\end{aligned}
$$


(in fact, in [V] there is an additional term defined by an integral over the boundary which is equal to zero in our case since $\partial D(0, R)$ is the circle). We then have

$$
|P f(z)-P f(0)| \leq|z| I_{1}+|z| I_{2}+\pi|f(z)|,
$$

where

$$
I_{1}=\iint_{D(0, R)} \frac{|f(\zeta)-f(z)|}{|\zeta-z|^{2}|\zeta|} d \xi d \eta \quad \text { and } \quad I_{2}=\iint_{D(0, R)} \frac{|f(\zeta)|}{|\zeta|^{2}|\zeta-z|} d \xi d \eta
$$

Since $f \in E_{R}$, to prove the lemma, we need only to show that

$$
\lim _{z \rightarrow 0}|z| I_{k} \log ^{q} \frac{1}{|z|}=0 \quad \text { for } \quad k=1,2 .
$$

For $k=2$, (4.31) holds by Lemma 4.1. To prove it for $k=1$, notice that since $f \in E_{R}$, then

$$
|f(\zeta)-f(z)|=o\left(\log ^{-q} \frac{1}{|\zeta-z|}\right) \quad \forall q>0
$$

uniformly in $z$. Hence for $|z|<\frac{r}{2}$, we have

$$
I_{1}=\iint_{D(z, R)} \frac{f(\tau+z)-f(z)}{|\tau|^{2}|\tau+z|} d s d t \leq \iint_{D(0,2 R)} \frac{|h(\tau, z)|}{|\tau|^{2}|\tau+z|} d s d t,
$$

where we have set $\tau=s+i t$ and $h(\tau, z)=f(z+\tau)-f(z)$. It follows from (4.32) that $h(., z) \in E_{R}$ and so (4.31) follows again from Lemma 4.1.

Theorem 4.2. Let $\mu(z) \in E_{R}$. The Beltrami equation

$$
\frac{\partial w}{\partial \bar{z}}=\mu(z) \frac{\partial w}{\partial z}
$$

has a solution of the form

$$
w(z)=z(1+K(z))
$$

with $K(z) \in E_{R}$.

Proof. It follows from classical results that any solution of (4.34) is $C^{\infty}$ away from 0 (for $R$ small enough) and it follows from [B] (Chapter 3) that equation (4.34) has a $C^{1}$ solution that is a local diffeomorphism at 0 . The local diffeomorphism can be constructed as follows (see [V], Chapter 2). Let

$$
w(z)=z+T f(z),
$$

with $f$ satisfying the integral equation

$$
f(z)-\mu(z) \Pi f(z)=\mu(z),
$$

where $T$ and $\Pi$ are the integral operators

$$
\begin{gathered}
T f(z)=\frac{-1}{\pi} \iint_{D(0, R)} \frac{f(\zeta)}{\zeta-z} d \xi d \eta, \\
\Pi f(z)=\frac{-1}{\pi} \iint_{D(0, R)} \frac{f(\zeta)}{(\zeta-z)^{2}} d \xi d \eta .
\end{gathered}
$$

Furthermore, the function $f$ is obtained as the limit of the sequence $f_{n}$ defined by

$$
f_{0}=0 \quad \text { and } \quad f_{n+1}(z)=\mu(z) \Pi f_{n}(z)+\mu(z) \quad \text { for } n \geq 0 .
$$


Each $f_{n}$ is in $E_{R}$ and so is $f$. The function

$$
v(z)=\frac{T f(z)-T f(0)}{z}=\frac{-1}{\pi} \iint_{D(0, R)} \frac{f(\zeta)}{\zeta(\zeta-z)} d \xi d \eta
$$

solves the equation

$$
\frac{\partial v}{\partial \bar{z}}=\frac{f}{z}
$$

It follows from Theorem 4.1 that

$$
K_{1}(z)=v(z)-v(0) \in E_{R} .
$$

Hence,

$$
w(z)=z+T f(z)-T f(0)
$$

has the desired form.

\section{A $C^{\infty}$ INTEGRAL}

We construct here a $C^{\infty}$ integral for $L_{n}$ defined in a tubular neighborhood of the characteristic circle. More precisely, we have the following theorem.

Theorem 5.1. Let $L_{n}$ be as in (2.1) and for $\delta>0$ let

$$
A_{\delta}=(-\delta, \delta) \times S^{1}, \quad A_{\delta}^{+}=(0, \delta) \times S^{1}, \quad A_{\delta}^{-}=(-\delta, 0) \times S^{1} .
$$

Then there is $\delta>0$ and $h \in C^{\infty}\left(A_{\delta}\right)$ such that

(८) $h$ is flat along the circle $\{r=0\}$;

(ıi ) $h: A_{\delta}^{ \pm} \longrightarrow h\left(A_{\delta}^{ \pm}\right)$is a diffeomorphism; and

(ıı ) $L_{n} h=0$.

The rest of the section deals with the proof of this theorem. Let

$$
\frac{P(r)}{r^{n}}+\mu \log |r|+i \theta+\sum_{j=1}^{\infty} f_{j}(\theta) r^{j}
$$

be the series constructed in Section 2, where $P$ is the polynomial of degree $\leq n-1$ given by

$$
P(r)=\alpha_{-n}+\alpha_{-n+1} r+\cdots+\alpha_{-1} r^{n-1} .
$$

Note that

$$
P(0)=\frac{-1}{n c_{0}}=\frac{-1}{n(1+i \beta)} \quad \text { and } \quad \operatorname{Re}(P(0))=\frac{-1}{n\left(1+\beta^{2}\right)}<0 .
$$

Let $g(r, \theta) \in C^{\infty}\left(A_{\delta}\right)$ be such that

$$
\frac{\partial^{j} g}{\partial r^{j}}(0, \theta)=j ! f_{j}(\theta), \quad \forall j \in \mathbb{Z}^{+} .
$$

Thus the Taylor series of $g$ with respect to $r$ is $\sum f_{j}(\theta) r^{j}$. Let

$$
m(r, \theta)=\frac{P(r)}{r^{n}}+\mu \log |r|+i \theta+g(r, \theta) .
$$

The function $m$ is $C^{\infty}$ in $\mathbb{R} \times \mathbb{R}$ for $r \neq 0$ small, and it satisfies

$$
m(r, \theta+2 \pi)=m(r, \theta)+2 \pi \quad \forall(r, \theta) .
$$


It follows from Proposition 2.1 and from (5.5) that $L_{n} m$ is flat along $r=0$. That is,

$$
L_{n} m(r, \theta)=o\left(r^{q}\right) \quad \forall q>0 .
$$

Define a function $f \in C^{\infty}\left(A_{\delta}\right)$ by

$$
f(r, \theta)=\exp \left(\epsilon(r)^{n} m(r, \theta)\right),
$$

where $\epsilon(r)=\frac{r}{|r|}$. The function $f$ satisfies

$$
|f(r, \theta)|=0\left(\exp \left(\frac{\operatorname{Re}(P(0))}{|r|^{n}}\right)\right) .
$$

Consequently, $f$ vanishes to infinite order along $r=0$ since $\operatorname{Re}(P(0))<0$.

Lemma 5.1. There exists $\delta>0$ such that the maps

$$
f: A_{\delta}^{ \pm} \longrightarrow f\left(A_{\delta}^{ \pm}\right),
$$

defined in (5.9), are diffeomorphisms.

Proof. We will prove the lemma for $A_{\delta}^{+}$. We need only to show that there exists $\delta>0$ for which $f$ is injective in $A_{\delta}^{+}$. Consider the equation

$$
f(r, \theta)=f(\rho, \phi) .
$$

By equating the real and imaginary parts we obtain

$$
\begin{gathered}
\frac{P_{1}(r)+\mu_{1} r^{n} \log r+r^{n} g_{1}(r, \theta)}{r^{n}}=\frac{P_{1}(\rho)+\mu_{1} \rho^{n} \log \rho+\rho^{n} g_{1}(\rho, \phi)}{\rho^{n}}, \\
\theta+\frac{P_{2}(r)+\mu_{2} r^{n} \log r+r^{n} g_{2}(r, \theta)}{r^{n}}=\phi+\frac{P_{2}(\rho)+\mu_{2} \rho^{n} \log \rho+\rho^{n} g_{2}(\rho, \phi)}{\rho^{n}}
\end{gathered}
$$

where we have set

$$
\mu=\mu_{1}+i \mu_{2}, \quad P=P_{1}+i P_{2}, \quad \text { and } \quad g=g_{1}+i g_{2} .
$$

The first equation of (5.13) can be rewritten as

$$
\frac{r}{\sqrt[n]{-P_{1}(r)-\mu_{1} r^{n} \log r-r^{n} g_{1}(r, \theta)}}=\frac{\rho}{\sqrt[n]{-P_{1}(\rho)-\mu_{1} \rho^{n} \log \rho-\rho^{n} g_{1}(\rho, \phi)}} .
$$

We have then, from the implicit function theorem, that for $\delta$ small enough (5.13) has a solution of the form

$$
\rho=r(1+r \alpha(r, \theta)) \text { and } \phi=\theta+r \beta(r, \theta) .
$$

Now, it can be proved that $f$ is a local diffeomorphism in a neighborhood of each point $(r, \theta)$ with $r \neq 0$. This, together with (5.15), imply that the functions $\alpha$ and $\beta$ are identically zero and so $f$ is injective on $A_{\delta}^{+}$. A similar argument shows that $f$ is also injective on $A_{\delta}^{-}$.

Since $f$ is flat along $r=0$, it follows from Lemma 5.1 that there exists $R=R(\delta)$ such that

$$
(D(0, R) \backslash 0) \subset f\left(A_{\delta}^{ \pm}\right) \subset \mathbb{C} .
$$

Let

$$
L^{ \pm}=f_{*} L_{n}
$$

be the pushforward to $f\left(A_{\delta}^{ \pm}\right)$of the vector field $L_{n}$ via $f$. 
Lemma 5.2. There exist a function $A(z) \in E_{R}$, where $E_{R}$ is the space of functions defined in (4.1), and a function $B(z)$ with

$$
B \in C^{\infty}(D(0, R) \backslash 0) \quad \text { and } \quad B(z) \log ^{1 / n}\left(\frac{1}{|z|}\right) \text { is bounded }
$$

such that the vector field $L^{+}$defined by (5.17) can be expressed as

$$
L^{+}=z A(z) \frac{\partial}{\partial z}-\frac{2 i}{\overline{c_{0}}} \bar{z}(1+B(z)) \frac{\partial}{\partial \bar{z}} .
$$

A similar expression holds for $L^{-}$.

Proof. Since $z=f(r, \theta)$, then it follows from (5.10) that there exist positive constants $a$ and $b$ such that

$$
a \exp \left(-\left(\frac{\kappa}{r}\right)^{n}\right)<|z|<b \exp \left(-\left(\frac{\kappa}{r}\right)^{n}\right),
$$

where we have set $\kappa=\sqrt[n]{-P_{1}(0)}$. Equivalently,

$$
\kappa \log ^{-\frac{1}{n}} \frac{b}{|z|}<r<\kappa \log ^{-\frac{1}{n}} \frac{a}{|z|} .
$$

Let

$$
L^{+}=X(z) \frac{\partial}{\partial z}+Y(z) \frac{\partial}{\partial \bar{z}}
$$

where

$$
X(z)=\left(L_{n} f\right)\left(f^{-1}(z)\right) \quad \text { and } \quad Y(z)=\left(L_{n} \bar{f}\right)\left(f^{-1}(z)\right) .
$$

Using $f(r, \theta)=\exp m(r, \theta)$, we get

$$
L_{n} f=f L_{n} m \quad \text { and } \quad L_{n} \bar{f}=\bar{f} L_{n} \bar{m} .
$$

We know that $L_{n} m$ is flat along $r=0$, and

$$
\begin{aligned}
L_{n} \bar{m} & =\left(\frac{\partial}{\partial \theta}-i r^{n+1}\left(c_{0}+O(r)\right) \frac{\partial}{\partial r}\right)\left(\frac{\bar{P}(r)}{r^{n}}-i \theta+\bar{\mu} \log r+O(r)\right) \\
& =-i+i n c_{0} \bar{P}(0)+O(r)=\frac{-2 i}{\overline{c_{0}}}+O(r) .
\end{aligned}
$$

Hence, it follows from (5.22), (5.23) and (5.24) that

$$
X(z)=z A(z) \quad \text { and } \quad Y(z)=\frac{-2 i}{\overline{c_{0}}} \bar{z}(1+B(z)) .
$$

That $A \in E_{R}$ follows from (5.23), (5.20) and (5.8). That $B$ satisfies the conditions of the lemma follows from (5.22), (5.24), and (5.20).

We are going to construct a solution to the equation $L_{n} u=0$ in $A_{\delta}^{+}$in the form $u=f(r, \theta)(1+k(r, \theta))$, where $f$ is defined by (5.9) and where $k$ is a $C^{\infty}$ function vanishing to infinite order along $r=0$. The function $k$ will be defined as

$$
k(r, \theta)=K \circ f(r, \theta)
$$

where $K(z)$ is a solution of the equation

$$
L^{+}(z(1+K(z)))=0 \quad \text { in } \quad D(0, R),
$$

and where $L^{+}$is defined in (5.18). 
By using the expression of $\mathrm{L}^{+}$given in Lemma 5.2, we find that the function $U=\log (1+K)$ must solve the equation

$$
\frac{\partial U}{\partial \bar{z}}=\frac{M(z)}{\bar{z}}+\frac{z}{\bar{z}} M(z) \frac{\partial U}{\partial z},
$$

where we have set

$$
M(z)=\frac{\overline{c_{0}} A(z)}{2 i(1+B(z))} \in E_{R} .
$$

To solve (5.28), we first consider the Beltrami equation

$$
\frac{\partial w}{\partial \bar{z}}=\frac{z}{\bar{z}} M(z) \frac{\partial w}{\partial z} .
$$

Since this equation has a coefficient in $E_{R}$, then it follows from Theorem 4.2 that it has a solution $w$ of the form

$$
w(z)=z(1+s(z)) \quad \text { with } \quad s \in E_{R} .
$$

With respect to the new complex variable $w$, equation (5.28) becomes

$$
\frac{\partial U}{\partial \bar{z}}=\frac{N(w)}{\bar{w}},
$$

where

$$
N(w)=\frac{\bar{w} M}{\bar{z}\left(1-|M|^{2}\right) \overline{w_{z}}} .
$$

Hence, $N \in E_{R}$ and by Theorem 4.1, equation (5.32) has a solution $U(w) \in E_{R}$. The function

$$
K(z)=\exp (U(w(z)))-1 \in E_{R}
$$

solves (5.27) and consequently, the function $k(r, \theta)$ given by (5.26) is flat along $r=0$ (thanks to $(5.20)$ ) and

$$
L_{n}(f(r, \theta)(1+k(r, \theta)))=0 \quad \text { in } A_{\delta}^{+} .
$$

A similar argument gives a solution to the equation $L_{n} u=0$ in $A_{\delta}^{-}$of the form $u=f(1+\hat{k})$ with $\hat{k}$ flat along $r=0$. We define $h$ in $A_{\delta}$ by

$$
h(r, \theta)= \begin{cases}f(r, \theta)(1+k(r, \theta)) & \text { if } r \geq 0, \\ f(r, \theta)(1+\hat{k}(r, \theta)) & \text { if } r \leq 0 .\end{cases}
$$

It follows from the construction of $k$ and $\hat{k}$ that if $\delta$ is small enough, then $h$ satisfies all properties of Theorem 5.1. This completes the proof.

Remark 5.1. The integral $h(r, \theta)$ constructed above has the form

$$
h(r, \theta)=\exp \left(\epsilon(r)^{n}\left(\frac{P(r)}{r^{n}}+\mu \log |r|+i \theta+l(r, \theta)\right)\right)
$$

with $l \in C^{\infty}\left(A_{\delta}\right)$ and $l(0, \theta)=0$. In general, $l$ is not real analytic, even when $L_{n}$ is real analytic (see Section 3 ). 


\section{Normalization}

We make use of the first integral constructed in the previous section to find a normal form for the vector field $L_{n}$.

Theorem 6.1. Let $L_{n}$ be a vector field as in (2.1). Then there exists a unique polynomial $P(r)$ with $\operatorname{Re}(P(0))<0$ and of degree $\leq n-1$, and there exists a complex number $\mu$ such that $L_{n}$ is $C^{\infty}$-conjugate in a ring $A_{\delta}$ to the vector field

$$
R_{n}=\frac{\partial}{\partial \theta}-i \frac{r^{n+1}}{r P^{\prime}(r)-n P(r)+\mu r^{n}} \frac{\partial}{\partial r},
$$

with a $C^{\infty}$ integral given by

$$
f_{n}(r, \theta)=\exp \left(\epsilon(r)^{n}\left(\frac{P(r)}{r^{n}}+\mu \log |r|+i \theta\right)\right),
$$

where $\epsilon(r)=\frac{r}{|r|}$.

To prove the theorem, we use the first integral $h(r, \theta)$ given by (5.37). Our aim is to find new coordinates in which the function $l(r, \theta)$ is identically zero. Let

$$
A(r)=\frac{P(r)}{r^{n}}+i \mu \log |r| \text {. }
$$

We decompose the functions into their real and imaginary parts:

$$
A=A_{1}+i A_{2}, \quad P=P_{1}+i P_{2}, \quad l=l_{1}+i l_{2}, \quad \mu=\mu_{1}+i \mu_{2} .
$$

Lemma 6.1. The equation

$$
A_{1}(\rho)=A_{1}(r)+l_{1}(r, \theta)
$$

has a solution $\rho \in C^{\infty}\left(A_{\delta}\right)$ of the form

$$
\rho=r+r^{n+2} \beta(r, \theta) .
$$

Proof. Equation (6.5) can be rewritten as

$$
\frac{\rho}{\left(-P_{1}(\rho)-\rho^{n} \mu_{1} \log |\rho|\right)^{1 / n}}=\frac{r}{\left(-P_{1}(r)-r^{n} \mu_{1} \log |r|-r^{n} l_{1}(r, \theta)\right)^{1 / n}} .
$$

It follows at once from the implicit function theorem that (6.7) has a solution $\rho=r+o(r)$. We write this solution as $\rho=r(1+\alpha(r, \theta))$ and solve for the function $\alpha$. By rewriting (6.5) for the unknown $\alpha$, we get the equation

$$
G(r, \theta, \alpha)=0,
$$

where $G$ is a $C^{\infty}$ function defined for $|r|<\delta,|\alpha|<\delta$, and $\theta \in S^{1}$ by

$$
G(r, \theta, \alpha)=(1+\alpha)^{n}\left(P_{1}(r)+r^{n} l_{1}(r, \theta)\right)-P_{1}(r(1+\alpha))-\mu_{1} r^{n} \log (1+\alpha) .
$$

Since

$$
\frac{\partial G}{\partial \alpha}(0,0, \theta)=n P_{1}(0) \neq 0
$$

and

$$
\frac{\partial^{j} G}{\partial r^{j}}(0,0, \theta)=0 \quad \text { for } j=0, \cdots, n,
$$

the solution $\alpha$ satisfies $\alpha=o\left(r^{n}\right)$. This proves the lemma. 
Lemma 6.2. Let $\rho(r, \theta)$ be a function as in (6.6). Then

$$
\log r-\log \rho \in C^{\infty}\left(A_{\delta}\right) \quad \text { and } \quad \frac{P_{2}(r)}{r^{n}}-\frac{P_{2}(\rho)}{\rho^{n}} \in C^{\infty}\left(A_{\delta}\right), \theta 6.12
$$

where $P_{2}$ is the imaginary part of the polynomial $P$. Furthermore, the functions given in (6.12) vanish along $r=0$.

Proof. For $\rho$ as in (6.6), we have

$$
\log r-\log \rho=-\log \left(1+r^{n+1} \beta\right)
$$

which is clearly $C^{\infty}$ for $r$ small and vanishes for $r=0$. We also have

$$
\frac{P_{2}(r)}{r^{n}}-\frac{P_{2}(\rho)}{\rho^{n}}=\frac{\left(1+r^{n+1} \beta\right)^{n} P_{2}(r)-P_{2}\left(r\left(1+r^{n+1} \beta\right)\right)}{r^{n}\left(1+r^{n+1} \beta\right)^{n}} .
$$

Since

$$
\left(1+r^{n+1} \beta\right)^{n} P_{2}(r)-P_{2}\left(r\left(1+r^{n+1} \beta\right)\right)=o\left(r^{n+1}\right),
$$

the conclusions follow.

Proof of Theorem 6.1. Let $\rho$ be the solution (6.6) of equation (6.5). With respect to the coordinates $(\rho, \theta)$, the function $h(r, \theta)$ has the expression

$$
h(\rho, \theta)=\exp \left[\epsilon(\rho)^{n}\left(\frac{P_{1}(\rho)}{\rho^{n}}+\mu_{1} \log |\rho|+i\left(\theta+\frac{P_{2}(\rho)}{\rho^{n}}+\mu_{2} \log |\rho|+s(\rho, \theta)\right)\right)\right],
$$

where $s$ is given by

$$
s(\rho, \theta)=l_{2}(r, \theta)+\mu_{2}(\log |r|-\log |\rho|)+\frac{P_{2}(r)}{r^{n}}-\frac{P_{2}(\rho)}{\rho^{n}} .
$$

It follows from Lemma 6.2 that $s \in C^{\infty}\left(A_{\delta}\right)$ and that $s=0$ along $\rho=0$. Finally, if we take as a new angle,

$$
\phi=\theta+s(\rho, \theta),
$$

then with respect to the new coordinates $(\rho, \phi)$, the function $h$ has the desired form

$$
h(\rho, \theta)=\exp \left(\epsilon(\rho)^{n}(A(\rho)+i \phi)\right)
$$

whose annihilator is the vector field $R_{n}(\rho, \phi)$ given in (6.1).

For a real analytic vector field $L_{n}$, the normal form $R_{n}$ can be achieved under a real analytic diffeomorphism only when the formal integral constructed in Section 2 converges for some $r \neq 0$. Under the assumption that the formal integral converges, the proof of the $C^{\omega}$-conjugacy is identical to that given above. We state this as the following theorem.

Theorem 6.2. Let $L_{n}$ be a real analytic vector field as in (2.1). Suppose that the corresponding formal solution converges for some $r \neq 0$. Then $L_{n}$ is $C^{\omega}$-conjugate in a ring $A_{\delta}$ to the vector field

$$
R_{n}=\frac{\partial}{\partial \theta}-i \frac{r^{n+1}}{r P^{\prime}(r)-n P(r)+\mu r^{n}} \frac{\partial}{\partial r} .
$$




\section{THE KERNEL OF $R_{n}$}

We determine the structure of the solutions of the homogeneous equation

$$
R_{n} u=0
$$

in the ring $A_{\delta}=(-\delta, \delta) \times S^{1}$.

Theorem 7.1. Let $f_{n}$ be the first integral given in (6.2) of the vector field $R_{n}$. A function $u \in C^{0}\left(\overline{A_{\delta}}\right)$ solves (7.1) if and only if there exist holomorphic functions $H^{ \pm}$defined in a neighborhood of $0 \in \mathbb{C}$, with $H^{+}(0)=H^{-}(0)$, such that

$$
u(r, \theta)=H^{ \pm} \circ f_{n}(r, \theta) \quad \forall(r, \theta) \in \overline{A_{\delta}^{ \pm}},
$$

where $A_{\delta}^{+}=A_{\delta} \cap\{r>0\}$ and $A_{\delta}^{-}=A_{\delta} \cap\{r<0\}$. Consequently, any $C^{0}$-solution of (7.1) is $C^{\infty}$.

Proof. The pushforward of $u$ in $A_{\delta}^{+}$via the first integral $f_{n}$ is a function $H^{+}$defined in $f_{n}\left(A_{\delta}^{+}\right)$that satisfies the CR equation $H_{\bar{z}}^{+}=0$. Hence, $H^{+}$is a bounded holomorphic function defined in a neighborhood of $0 \in \mathbb{C}$. Therefore, $u=H^{+} \circ f_{n}$ in $A_{\delta}^{+}$. A similar result holds in $A_{\delta}^{-}$. That $H^{+}(0)=H^{-}(0)$ follows from the continuity of $u$ and that $u$ is $C^{\infty}$ on $r=0$ follows from the flatness of $f_{n}$ along $r=0$.

Remark 7.1. Theorem 7.1 does not have a local counterpart version. For every $p \in \Sigma$ there exist $C^{0}$ solutions of $L_{n} u=0$ defined in a neighborhood of $p$ that are not $C^{\infty}$. For example, for a given branch of the logarithm, the function $\left(x+i x^{2} t\right)^{3 / 2}$ is not $C^{\infty}$ in a neighborhood of 0 and it satisfies the equation

$$
\left(\frac{\partial}{\partial t}-i \frac{x^{2}}{1+2 i x t} \frac{\partial}{\partial x}\right) u=0
$$

(we refer to [T1] and [T2] for the local solvabilty of vector fields).

The next result describes the distribution solutions of (7.1) that are supported by the characteristic circle $r=0$. The analogue question for the vector field $L_{0}$ is treated in BhM2].

Theorem 7.2. Let $u \in \mathcal{D}^{\prime}\left(A_{\delta}\right)$ with $\operatorname{supp}(u) \subset\{r=0\}$. If $u$ solves (7.1), then there exist constants $c_{0}, \cdots, c_{n-1}$ in $\mathbb{C}$ such that

$$
\langle u, \phi\rangle=\sum_{j=0}^{n-1} c_{j} \int_{0}^{2 \pi} \frac{\partial^{j} \phi}{\partial r^{j}}(0, \theta) d \theta \quad \forall \phi \in \mathcal{D}\left(A_{\delta}\right) .
$$

Proof. The transpose of $R_{n}$ is the operator

$$
R_{n}^{*}=-\frac{\partial}{\partial \theta}+i r^{n+1} Q(r) \frac{\partial}{\partial r}+i\left(r^{n+1} Q(r)\right)_{r},
$$

where

$$
Q(r)=\frac{1}{r P^{\prime}(r)-n P(r)+\mu r^{n}} .
$$

First, we verify that a distribution $u$ given by (7.3) solves equation (7.1). For $j=0, \cdots, n-1$, let $u_{j} \in \mathcal{D}^{\prime}\left(A_{\delta}\right)$ be defined by

$$
\left\langle u_{j}, \phi\right\rangle=\int_{0}^{2 \pi} \frac{\partial^{j} \phi}{\partial r^{j}}(0, \theta) d \theta \quad \forall \phi \in \mathcal{D}\left(A_{\delta}\right) .
$$


For $\phi \in \mathcal{D}\left(A_{\delta}\right)$, we write

$$
\phi(r, \theta)=\sum_{k=0}^{n-1} l_{k}(\theta) r^{k}+O\left(r^{n}\right), \quad l_{k}(\theta) \in C^{\infty}\left(S^{1}\right) .
$$

It follows that

$$
R_{n}^{*} \phi=-\sum_{k=0}^{n-1} l_{k}^{\prime}(\theta) r^{k}+O\left(r^{n}\right)
$$

Therefore,

$$
\frac{\partial^{j} R_{n}^{*} \phi}{\partial r^{j}}(0, \theta)=-j ! l_{j}^{\prime}(\theta)
$$

and

$$
\left\langle R_{n} u_{j}, \phi\right\rangle=\left\langle u_{j}, R_{n}^{*} \phi\right\rangle=\int_{0}^{2 \pi} \frac{\partial^{j} R_{n}^{*} \phi}{\partial r^{j}}(0, \theta) d \theta=-j ! \int_{0}^{2 \pi} l_{j}^{\prime}(\theta) d \theta=0 .
$$

Hence, $u_{j}$ solves (7.1) and so does any linear combination given by (7.3).

Let $u \in \mathcal{D}^{\prime}\left(A_{\delta}\right)$ be a solution of $(7.1)$ and $\operatorname{supp}(u) \subset\{r=0\}$. Suppose that $u$ has a transverse order $m$. Since $R_{n}$ is elliptic in the tangential direction along $r=0$, then there exist $a_{0}(\theta), \cdots, a_{m}(\theta) \in C^{\infty}\left(S^{1}\right)$ such that

$$
\langle u, \phi\rangle=\sum_{k=0}^{m} \int_{0}^{2 \pi} a_{k}(\theta) \frac{\partial^{k} \phi}{\partial r^{k}}(0, \theta) d \theta .
$$

We prove that the order $m$ must satisfy $m \leq n-1$. By contradiction, suppose that $m \geq n$. Then $a_{m} \neq 0$ and there exists $p \in \mathbb{Z}$ such that

$$
\int_{0}^{2 \pi} a_{m}(\theta) \mathrm{e}^{i p \theta} d \theta \neq 0
$$

If $p \neq 0$, we let $\phi \in \mathcal{D}\left(A_{\delta}\right)$ be of the form

$$
\phi(r, \theta)=\mathrm{e}^{i p \theta} r^{m}+o\left(r^{m}\right) .
$$

Then

$$
R_{n}^{*} \phi=-i p \mathrm{e}^{i p \theta} r^{m}+o\left(r^{m}\right)
$$

and

$$
\begin{aligned}
\frac{\partial^{j} R_{n}^{*} \phi}{\partial r^{j}}(0, \theta) & =0 \quad \text { if } j=0, \ldots, m-1, \\
\frac{\partial^{m} R_{n}^{*} \phi}{\partial r^{m}}(0, \theta) & =-m ! i p \mathrm{e}^{i p \theta} .
\end{aligned}
$$

It follows from (7.10), (7.11) and (7.14) that

$$
\left\langle R_{n} u, \phi\right\rangle=\left\langle u, R_{n}^{*} \phi\right\rangle=\int_{0}^{2 \pi} a_{m}(\theta)\left(-m ! i p \mathrm{e}^{i p \theta}\right) d \theta \neq 0 .
$$

This contradicts the hypothesis $R_{n} u=0$ and shows that $m \leq n-1$ when $p \neq 0$. In the case $p=0$, we consider $\phi \in \mathcal{D}\left(A_{\delta}\right)$ independent of $\theta$ and given by

$$
\phi(r, \theta)=g(r)=r^{m-n}+o\left(r^{m-n}\right)
$$

with $g \in \mathcal{D}((-\delta, \delta))$. We have (by using (7.4)) that

$$
R_{n}^{*} \phi(r, \theta)=i \frac{d}{d r}\left(r^{n+1} Q(r) g(r)\right)=i(m+1) Q(0) r^{m}+o\left(r^{m}\right) .
$$


Consequently,

$$
\begin{gathered}
\frac{\partial^{j} R_{n}^{*} g}{\partial r^{j}}(0, \theta)=0 \quad \text { if } j=0, \ldots, m-1, \\
\frac{\partial^{m} R_{n}^{*} \phi}{\partial r^{m}}(0, \theta)=i(m+1) ! Q(0) \neq 0 .
\end{gathered}
$$

A similar argument shows that in this case, we also have $\left\langle R_{n} u, g\right\rangle \neq 0$. This shows that the order of a distribution solution supported by the characteristic circle needs to be $\leq n-1$.

Next, we prove that the coefficients $a_{0}(\theta), \cdots, a_{m}(\theta)$ given in (7.10) are constants. For $\phi \in \mathcal{D}\left(A_{\delta}\right)$, we have

$$
0=\left\langle R_{n} u, \phi\right\rangle=\sum_{j=0}^{m} \int_{0}^{2 \pi} a_{j}(\theta) \frac{\partial^{j} R_{n}^{*} \phi}{\partial r^{j}}(0, \theta) d \theta .
$$

We write

$$
\phi(r, \theta)=\sum_{k=0}^{m} l_{k}(\theta) r^{k}+o\left(r^{m}\right) .
$$

Since $m<n$, it follows from (7.4) and (7.20) that for $j=0, \cdots, m$,

$$
\frac{\partial^{j} R_{n}^{*} \phi}{\partial r^{j}}(0, \theta)=j ! l_{j}^{\prime}(\theta) \text {. }
$$

For a given $k \leq m$, let $\phi_{k} \in \mathcal{D}^{\prime}\left(A_{\delta}\right)$ be such that

$$
\phi_{k}(r, \theta)=l_{k}(\theta) r^{k}+o\left(r^{m}\right) .
$$

Equation (7.19) together with (7.21) gives

$$
0=\left\langle R_{n} u, \phi_{k}\right\rangle=k ! \int_{0}^{2 \pi} a_{k}(\theta) l_{k}^{\prime}(\theta) d \theta .
$$

If $a_{k}$ were not constant, then there would be $p \in \mathbb{Z}$ with $p \neq 0$ such that

$$
\int_{0}^{2 \pi} a_{k}(\theta) \mathrm{e}^{i p \theta} d \theta \neq 0
$$

and in this case if we select $f_{k}(\theta)=\mathrm{e}^{i p \theta}$, then (7.23) will be violated. This shows that $a_{0}, \cdots, a_{m}$ are constants.

\section{A degenerate Beltrami equation}

The Beltrami equation $w_{\bar{z}}=\mu(z) w_{z}$ has been studied in the elliptic case $|\mu(z)| \leq$ $K<1$ for all $z$ in a domain of $\mathbb{C}$ (see $[\mathrm{V}]$ ). However, very little is known when $\mu(z)$ is not uniformly bounded away from 1 . In this section, we consider this degenerate situation and show that it can be understood in terms of the vector field $L_{n}$ with $n=0$.

We start with a vector field $V$ defined in a disc $D(0, \delta) \subset \mathbb{C}$ by

$$
V=A(z) \frac{\partial}{\partial z}+B(z) \frac{\partial}{\partial \bar{z}}
$$

where $A, B \in C^{l}(D(0, R))$ satisfy

$$
|A(z)|=O\left(|z|^{m}\right) \quad \text { and } \quad|B(z)|=O\left(|z|^{n}\right)
$$


with $m, n \in \mathbb{Z}^{+}$such that

$$
n \leq m<l .
$$

Assume that there exist constants $a, b>0$ such that

$$
a|z|^{2 n} \leq|B(z)|^{2}-|A(z)|^{2} \leq b|z|^{2 n} .
$$

The equation $V w=0$ is equivalent to the Beltrami equation

$$
w_{\bar{z}}=\mu(z) w_{z},
$$

with

$$
\mu(z)=-\frac{A(z)}{B(z)} .
$$

It follows from hypothesis (8.4) that

$$
|\mu(z)|<1 \quad \text { for } z \neq 0 .
$$

Hence, equation (8.5) is elliptic in a neighborhood of each point $z \neq 0$, but $\lim \sup _{z \rightarrow 0}|\mu(z)|$ might be equal to 1 . We will show that this degenerate Beltrami equation has a solution which is a local homeomorphism at 0 .

Theorem 8.1. Let $\mu(z)$ be given by (8.6) with $A$ and $B$ satisfying (8.4). Then there exist $\delta>0, \sigma>0$ and a function

$$
w \in C^{l+1}(D(0, R) \backslash 0) \cap C^{\sigma}(D(0, R))
$$

such that $w$ solves the Beltrami equation (8.5) and

$$
w: D(0, R) \longrightarrow w(D(0, R))
$$

is a homeomorphism.

Proof. First, consider the case $m>n$. It follows from the hypotheses that

$$
\mu(z) \in C^{l}(D(0, R) \backslash 0) \cap C^{m-n-1+\sigma}(D(0, R))
$$

for any $0<\sigma<1$ and that $\mu(0)=0$. This is a classical Beltrami equation and a diffeomorphic solution $w$ can be found in $D(0, R)$. With $w$ of class $C^{l+1}$ away from 0 , and of class $C^{m-n+\sigma}$ at 0 (see $[\mathrm{V}]$ ).

Next, in the case $m=n$, in which there is an effective degeneracy, let

$$
\begin{aligned}
& A(z)=A_{n}(z)+O\left(|z|^{n+1}\right), \\
& B(z)=B_{n}(z)+O\left(|z|^{n+1}\right)
\end{aligned}
$$

with $A_{n}$ and $B_{n}$ homogeneous polynomials in $z$ and $\bar{z}$ of degree $n$. We use polar coordinates $z=r \mathrm{e}^{i \theta}$ to express (8.4) as

$$
a \leq\left|B\left(\mathrm{e}^{i \theta}\right)\right|^{2}-\left|A\left(\mathrm{e}^{i \theta}\right)\right|^{2} \leq b
$$

and the vector field $V$ as

$$
V=\frac{i}{2} r^{n-1}\left(B_{n}\left(\mathrm{e}^{i \theta}\right) \mathrm{e}^{i \theta}-A_{n}\left(\mathrm{e}^{i \theta}\right) \mathrm{e}^{-i \theta}+O(r)\right)\left(\frac{\partial}{\partial \theta}-i r(a(\theta)+O(r)) \frac{\partial}{\partial r}\right),
$$

where

$$
a(\theta)=\frac{B_{n}\left(\mathrm{e}^{i \theta}\right) \mathrm{e}^{i \theta}+A_{n}\left(\mathrm{e}^{i \theta}\right) \mathrm{e}^{-i \theta}}{B_{n}\left(\mathrm{e}^{i \theta}\right) \mathrm{e}^{i \theta}-A_{n}\left(\mathrm{e}^{i \theta}\right) \mathrm{e}^{-i \theta}}
$$


It follows from (8.12) that the real part of $a(\theta)$ is nowhere zero. We can therefore assume that $\operatorname{Re}(a)>0$ so that

$$
\lambda=\frac{1}{2 \pi} \int_{0}^{2 \pi} a(\theta) d \theta \in \mathbb{R}^{+}+i \mathbb{R} .
$$

It follows then from M2 that there exists $\delta>0$ such that the equation $V u=0$ has a solution of the form

$$
u(r, \theta)=r^{1 / \lambda} \mathrm{e}^{i \theta} B(r, \theta)
$$

with

$$
B \in C^{l+1}\left(A_{\delta} \backslash\{r=0\}\right) \cup C^{0}\left(A_{\delta}\right)
$$

and $B(0, \theta) \neq 0$ for every $\theta$. Hence, for $\delta$ small enough $u$ is a homeomorphism from $A_{\delta}^{+}$onto its image $u\left(A_{\delta}^{+}\right)$. The expression of the function $u$ in terms of the variable $z=r \mathrm{e}^{i \theta}$ is

$$
w(z)=\frac{z}{|z|}|z|^{1 / \lambda} \hat{B}(Z)
$$

with $\hat{B}(z)=B\left(u^{-1}(z)\right)$. Thus

$$
w \in C^{l}(D(0, \epsilon) \backslash 0) \cap C^{\sigma}(D(0, \epsilon))
$$

for any positive number $\sigma$ satisfying

$$
0<\sigma<\operatorname{Re}\left(\frac{1}{\lambda}\right)
$$

Furthermore, $w$ is a homeomorphism and satisfies equation (8.5).

As a consequence of Theorem 8.1 we get the following factorization result.

Theorem 8.2. If $u$ is a $C^{0}$-solution of (8.5) defined near $0 \in \mathbb{C}$, then there exists a holomorphic $H$ such that $u=H \circ w$, where $w$ is the homeomorphic solution of (8.5) as in Theorem 8.1.

Remark 8.1. The following question (motivated by geometric considerations) is considered in [W] (page 52). Given $A(x, y)$ and $B(x, y)$ real analytic functions defined near $0 \in \mathbb{R}^{2}$ such that

$$
\left|\frac{A(x, y)}{B(x, y)}\right| \leq K<1 \quad \text { for } \quad x^{2}+y^{2} \leq R^{2},
$$

the question is to determine whether the Beltrami equation $w_{\bar{z}}=(A / B) w_{z}$ has a meromorphic solution. That is, a solution of the form

$$
w(x, y)=\frac{f(x, y)}{g(x, y)}
$$

with $w$ a local homeomorphism at 0 and $f$ and $g$ real analytic. In view of Theorem 8.1 and its proof, in general, there are no nontrivial meromorphic solutions to such Beltrami equations. Indeed, a necessary condition for the existence of a meromorphic solution is that the invariant $\lambda$ (see (8.15)) of the associated vector field $V$ (as in (8.13)) must be in $\mathbb{Z}^{+}$. This follows from the fact that if the solution is meromorphic, then its expression in polar coordinates $(r, \theta)$ would be a real 
analytic integral of $V$ and thus $\lambda \in \mathbb{Z}^{+}$(see [M1] and [M2]). However, for given real analytic functions $A, B$, the associated invariant $\lambda$ is not necessarily in $\mathbb{Z}$. In fact, even when $\lambda \in \mathbb{Z}^{+}$, there are vector fields without nontrivial $C^{\omega}$ solutions (see [CG]).

\section{REFERENCES}

[BCH] A. Bergamasco, P. Cordaro, and J. Hounie, Global properties of a class of vector fields in the plane, J. Diff. Equations 74 (1988), 179-199. MR89h:58175

[BCP] A. Bergamasco, P. Cordaro, and G. Petronilho, Global Solvability for a class of complex vector fields on the two-torus, Preprint.

[BgM1] A.Bergamasco and A. Meziani, Semiglobal solvability of a class of planar vector fields of infinite type, Mat. Contemporanea 18 (2000), 31-42. MR2001m:35013

[BgM2] A.Bergamasco and A. Meziani, Solvability near the characteristic set for a class of planar vector fields of infinite type, Preprint.

[BhM1] S. Berhanu and A. Meziani, On rotationally invariant vector fields in the plane, Manus. Math. 89 (1996), 355-371. MR.97e:35007

[BhM2] S. Berhanu and A. Meziani, Global properties of a class of planar vector fields of infinite type, Comm. PDE 22 (1997), 99-142. MF98c:35003

[B] N. Bliev, Generalized analytic functions in fractional spaces, Pitman Monographs and Surveys in Pure and Applied Mathematics 86, 1997. MR.98g:30075

[CG] P. Cordaro and X. Gong, Normalization of complex-valued planar vector fields which degenerate along a real curve, Preprint.

[M1] A. Meziani, On real analytic planar vector fields near the characteristic set, Contemp. Math. 251 (2000), 429-438. MR.2001e:35033

[M2] A. Meziani, On planar elliptic structures with infinite type degeneracy, J. Funct. An. 179 (2001), 333-373. MR2001k:35122

[NT] L. Nirenberg and F. Treves, Solvability of first order pde, Comm. Pure Applied Math. 16 (1963), 331-351. MR 29:348

[T1] F. Treves, Remarks about certain first-order linear PDE in two variables, Comm. PDE 5 (1980), 381-425. MR.83e:35033

[T2] F. Treves, Hypo-analytic structures: local theory, Princeton Univ. Press, 1992. MR.94e:35014

[V] I. Vekua, Generalized analytic functions, Pergamon Press, 1962. MR27:321

[W] W. L. Wendland, Elliptic systems in the plane, Pitman Monographs and Studies in Mathematics 3, 1979. MR80h:35053

Department of Mathematics, Florida International University, Miami, Florida 33199

E-mail address: meziani@fiu.edu 